\title{
Visualising reporting patterns of Citizen Science observations and their importance for the interpretation of data and visualisations: the example of ornithological observations
}

\author{
Jana Moser ${ }^{\mathrm{a}, *}$, Johannes Wahl ${ }^{\mathrm{b}}$, Stephan Schwan ${ }^{\mathrm{c}}$, Julia Moritz ${ }^{\mathrm{c}}$, Tom Hoyer ${ }^{\mathrm{a}}$, Sebastian Geidel ${ }^{\mathrm{a}}$, \\ Christopher König ${ }^{b}$ \\ ${ }^{a}$ Leibniz-Institut für Länderkunde, Germany,Jana Moser-j_moser@leibniz-ifl.de,Tom Hoyer-t_hoyer@leibniz-ifl.de, Sebastian \\ Geidel-s_geidel@leibniz-ifl.de \\ ${ }^{b}$ Dachverband Deutscher Avifaunisten e.V., Germany, JohannesWahl-johannes.wahl@dda-web.de, Christopher König - \\ christopher.koenig@dda-web.de \\ ${ }^{c}$ Leibniz-Institut für Wissensmedien, Germany, Stephan Schwan-s.schwan@iwm-tuebingen.de, Julia Moritz-j.moritz@iwm- \\ tuebingen.de \\ * Corresponding author
}

Keywords: crowd-mapping, visualisation, Citizen Science, participation, usability studies

\begin{abstract}
:
Every day, nature enthusiasts collect valuable information about local animal and plant populations. This has resulted in approximately 50 million data sets until now for the bird observations at ornitho.de alone, with the observation numbers having risen sharply during the last three years. Such data may not always meet all scientific criteria, but due to their quantity they allow statements to be made, e.g. about regional distribution patterns or local occurrences. However, the citizens involved in the survey and the interested public are still unable to use these data, or can only use them in very low resolution or at great effort. According to the current Citizen Science Guidelines, data collecting citizens and other interested parties should be able to use such data and, for example, discover the ornithological diversity on their own doorstep. For this reason, the "Experiencing biodiversity" project has developed web maps, which, by the way, should inspire even more people to record biodiversity.
\end{abstract}

The presentation will firstly address the visualization and interpretation of citizen science data collections. In order to create awareness that numerous animal and plant species are in danger and need special protection, an overview of the current species inventory is necessary. The aim of the project is to provide this overview by visual means to the participating citizen scientists but also to the interested public. The maps should be easy to understand and take into account the varying degrees of citizen science participation in different regions. Thus, in addition to the occurrence of a bird species, the reporting activity is important and therefore displayed on the map. This is unique and shows how many reports are available for all bird species recorded. Thus, it is possible to estimate the amount and distribution of the available data and to compare them with the reported occurrence of a single bird species. As a result, data gaps or changes over time become visible and support the interpretation of the data. As there are no negative reports, only this additional level allows an interpretation at all whether a bird species is "not present" or whether it was just "not reported" in certain areas. At the same time, the project wants to contribute to a better understanding of the visualizations, but also of the underlying data basis.

Secondly, the presentation will also address the sometimes contradictory relationship between visualization - i.e. making visible - and the most diverse interests of protection - i.e. avoiding making visible. And thirdly, challenges in the visualization of mass data in the sense of a performant presentation of such data to a broad public will be discussed. A special challenge with regard to performance issues are rapidly increasing data volumes and questions of data protection, data transfer to the visualization but also permanent costs for the provision of data which should not be underestimated.

Due to the ongoing status of the project, interim results are presented and mirrored with currently ongoing scientific studies on the use of the maps by Citizen Scientists and the influence of the visualisations on the motivation of ornithologists. Advantages and disadvantages of this visualisation will be discussed with regard to its transferability to other projects. 